\title{
Real Perturbation of Complex Analytic Families: Points to Regions
}

\author{
Bruce B. Peckham \\ Department of Mathematics and Statistics \\ University of Minnesota, Duluth \\ Duluth, Minnesota 55812 \\ bpeckham@d.umn.edu
}

July 15, 2002

\begin{abstract}
This study provides some connections between bifurcations of onecomplex-parameter complex analytic families of maps of the complex plane $\mathcal{C}$ and bifurcations of more general two-real-parameter families of real analytic (or $C^{k}$ or $C^{\infty}$ ) maps of the real plane $\mathcal{R}^{2}$. We perform a numerical study of local bifurcations in the families of maps of the plane given by

$$
z \mapsto F_{(C, \alpha)}(z, \bar{z})=z^{2}+C+\alpha \bar{z}
$$

where $z$ is a complex dynamic (phase) variable, $\bar{z}$ its complex conjugate, $C$ is a complex parameter, and $\alpha$ is a real parameter. For $\alpha=0$, the resulting family is the familiar complex quadratic family. For $\alpha \neq 0$, the map fails to be complex analytic, but is still analytic (quadratic) when viewed as a map of $\mathcal{R}^{2}$. We treat $\alpha$ in this family as a perturbing parameter and ask how the two-parameter bifurcation diagrams in the $C$ parameter plane change as the perturbing parameter $\alpha$ is varied.

The most striking phenomenon that appears as $\alpha$ is varied is that bifurcation points in the $C$ plane for the quadratic family $(\alpha=0)$ evolve into fascinating bifurcation regions in the $C$ plane for nonzero $\alpha$. Such points are the cusp of the main cardioid of the Mandelbrot set and contact points between "bulbs" of the Mandelbrot set. Arnold resonance tongues are part of the evolved scenario.

We also provide sufficient conditions for more general perturbations of complex analytic maps of the plane of the form:

$$
z \mapsto F_{(C, \alpha)}(z, \bar{z})=f_{C}(z)+\alpha g_{\alpha}(z, \bar{z})
$$

to have bifurcation points for $\alpha=0$ which evolve into nontrivial bifurcation regions as $\alpha$ grows from zero.
\end{abstract}




\section{Introduction: Background and Motivation}

Complex analytic maps of the complex plane $\mathcal{C}$ form a very special subset in the more general function space of real analytic (or $C^{k}$ or $C^{\infty}$ ) maps of the real plane $\mathcal{R}^{2}$. Although the study of bifurcations of iterated maps in the two settings has overlap, the reliance on powerful tools in the complex analytic setting makes the two areas seem, at times, as completely distinct areas of research. In this paper, we provide a connection between the two areas by studying how bifurcation sets for a one-complex-parameter complex analytic family change when modified with a perturbation which is not complex analytic. See Drexler [1996] for a related study. See Bielefeld, Sutherland, Tangerman and Veerman [1993] for a study of a similar spirit - involving a different perturbation of the complex quadratic family but with some related results. It turns out that since this study and previous studies of forced oscillator systems [Peckham 1988, Vance and Ross 1989, Peckham 1990] both involve Hopf bifurcations, the bifurcation diagrams in the two settings have many similarities.

The model family we study is

$$
F_{(C, \alpha)}(z, \bar{z})=z^{2}+C+\alpha \bar{z}
$$

where $z$ is a complex dynamic (phase) variable, $\bar{z}$ its complex conjugate, $C=$ $C_{1}+i C_{2}$ is a complex parameter, and $\alpha$ is a real parameter. In cartesian coordinates, this is

$$
F_{\left(C_{1}, C_{2}, \alpha\right)}\left(\begin{array}{l}
x \\
y
\end{array}\right)=\left(\begin{array}{c}
x^{2}-y^{2}+C_{1}+\alpha x \\
2 x y+C_{2}-\alpha y
\end{array}\right) .
$$

The most striking phenomenon that occurs is that bifurcation points in the $C$ plane for the complex quadratic family ( $\alpha$ fixed at 0 ) evolve into fascinating bifurcation regions in the $C$ plane for small nonzero fixed $\alpha$. Such bifurcation points for the complex analytic family are cusps of cardioids of the Mandelbrot set and the contact points of neighboring "bulbs" in the Mandelbrot set. Look ahead to Fig. 4a for a typical nonzero $\alpha C$ plane bifurcation diagram. Compare it with the Mandelbrot set bulb boundaries of Fig. 2. This paper can largely be thought of as an attempt to understand these two figures and their relationship to each other.

In the next two subsections of the introduction, we recall basic facts about the bifurcations in generic one-complex-parameter families of complex analytic maps of the plane, including the complex quadratic family (subsection 1.1), and bifurcations in generic two-real-parameter families of maps of $\mathcal{R}^{2}$ (subsection 1.2). We then compare and contrast the two settings in subsection 1.3, emphasizing basic differences. Of particular note is the view of the bulb boundaries in the Mandelbrot set as Hopf bifurcation curves. Understanding the contrast between generic bifurcations emanating from a Hopf curve: bulb tangencies in the complex setting vs. Arnold resonance tongues in the real setting, was the original motivation behind this study. 
With the possible exception of subsection 1.1.3, all of the results stated in this Introduction are well-known. Most even exist in standard graduate textbooks [Arnold 1983, Devaney 1989, Guckenheimer and Holmes 1983, Ruelle 1989] or research surveys [Blanchard 1984]. They are included here to enable us to more easily compare and contrast the complex analytic dynamics with the real dynamics.

The new results in this paper are the details of the bifurcation diagrams in the perturbed cases (Sec. 2) and the theorems in Sec. 3. The theorems give sufficient conditions under which complex analytic bifurcation points evolve into bifurcation regions upon perturbation. They also give a characterization of some of the bifurcation sets that evolve.

\subsection{Complex analytic families: the Mandelbrot set and its bulbs}

We begin by recalling some definitions, properties, and bifurcations for the complex quadratic family

$$
Q_{C}(z) \equiv F_{(C, 0)}(z)=z^{2}+C .
$$

The local bifurcations of this classic family are expected to behave as generic bifurcations in one-complex-parameter families of complex analytic maps of the plane.

The most basic parameter plane "bifurcation diagram" for the complex quadratic family is the Mandelbrot set (Fig. 1), most easily defined as $\mathcal{M} \equiv\{c \in$ $\mathcal{C} \mid Q_{c}^{n}(0) \nrightarrow \infty$ as $\left.n \rightarrow \infty\right\}$. This set provides a (parameter space) dichotomy for all the of the complex quadratic maps. Maps corresponding to parameter values outside the Mandelbrot set all have similar dynamics. Maps corresponding to parameter values inside $\mathcal{M}$ also have similarities in their behaviors under iteration, but $\mathcal{M}$ is further subdivided into "bulbs," defined as the connected components of the interior of $\mathcal{M}$. All maps corresponding to parameter values in a single bulb of $\mathcal{M}$ have an attracting periodic orbit of the same period, say $n$. Thus these bulbs are often labelled according to their period as "period- $n$ bulbs."

The fixed-point bulb is the interior of the main cardioid of the Mandelbrot set. The period-two bulb is the disk bounded by a geometric circle with radius $1 / 4$ centered at $z=-1$. For $n>3$ there exist multiple bulbs of the same period. Except for the cusp of the main cardioid and the cardioids of all "baby Mandelbrot sets," all bulb boundaries are smooth.

Bifurcations thus occur when the parameter value is varied to either cross from inside to outside the Mandelbrot set (or vice versa) or as we pass from one bulb to another. Both cases involve passing through the boundary of a bulb. When crossing a period- $n$ bulb boundary, a period- $n$ orbit changes from being attracting for $C$ values inside a period- $n$ bulb to (linearly) neutral for $C$ values 


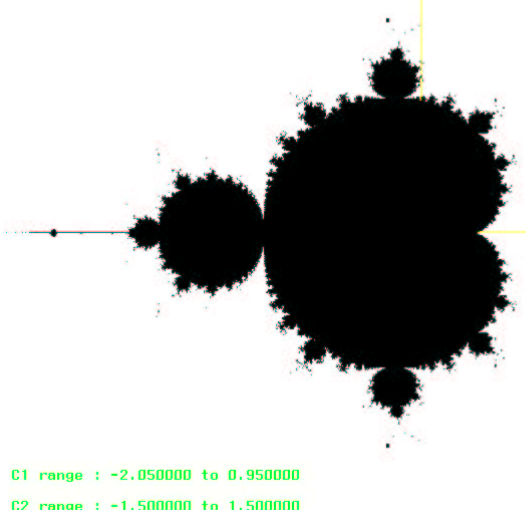

Figure 1: Aproximation of the Mandelbrot Set.

on the boundary of the bulb, to repelling for $C$ values outside the bulb. Thus, a point on the boundary of a period- $n$ bulb has a period- $n$ orbit with derivative on the unit circle. Moreover, when traveling once around the boundary of any period- $n$ bulb, the corresponding derivative travels once around the unit circle [Douady and Hubbard 1982]. By analogy with the real case, we shall refer to these bulb boundary curves as Hopf bifurcation curves.

Hopf bifurcation points are classified as rational or irrational. We deal mainly with the rational period $n$ bulb boundary point here $\left(f^{n^{\prime}}(z)=e^{2 \pi i p / q}\right.$, $p / q \in(0,1)$ reduced). At each such point is attatched a bulb of period $q n$. The bulb boundary points corresponding to a derivative of one fall into two classes: either there is a bulb attatched at the derivative one point or there isn't. In the latter case, the derivative one point is the cusp of a cardioid (either the main cardioid or a cardioid of a "baby Mandelbrot set"). In the former case, the attatched bulb is of lower period. In fact, at any bulb tangency, the lower period bulb is the parent, say period- $n$, and the higher period bulb its $p / q$ child for some $p / q \in(0,1)$. The child is then a period- $q$ n bulb. The contact point is a derivative $e^{2 \pi i p / q}$ point for the parent and a derivative one point for the child. We prefer to classify it as a $p / q$ point of the parent. The cardioids are the only bulbs without a parent. See Fig. 2 for a labelled picture of the main cardioid and some of its descendents. A $\left(\frac{p_{1}}{q_{1}}, \ldots, \frac{p_{k-1}}{q_{k-1}}, \frac{p_{k}}{q_{k}}\right)$ bulb is the $\frac{p_{k}}{q_{k}}$ child of the $\left(\frac{p_{1}}{q_{1}}, \ldots, \frac{p_{k-1}}{q_{k-1}}\right)$ bulb.

In preparation for comparison with the analogous bifurcations in subsection 1.2, we distinguish the eigenvalue one points (cardioid cusps) as well as the negative one eigenvalue points (period-doublings). 


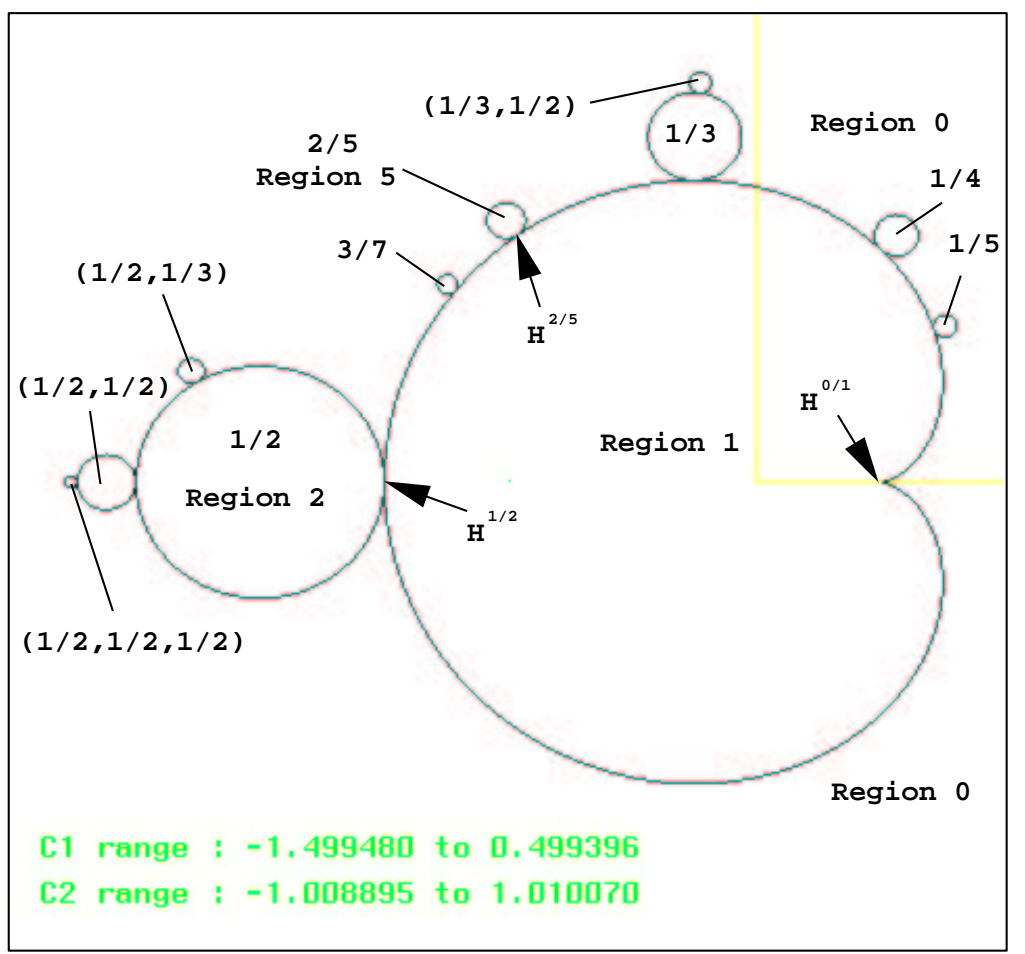

Figure 2: Some numerically computed bulb boundaries of which the Mandelbrot set boundary is comprised $(\alpha=0.0)$.

\subsubsection{Cardioid cusp points (saddle-node points)}

We look first at the bifurcation point at the cusp of the main (or any) cardioid. It is sometimes labelled a saddle-node because of the analogous bifurcation in the real plane, but the name is not at all descriptive of the complex bifurcation. (The name cusp point is also used in real bifurcation to indicate a saddle-node bifurcation with a higher order degeneracy; this is distinct from the cardioid cusp discussed here.) A cardioid cusp point requires a periodic point with eigenvalue one. The conditions which need to be satisfied are thus:

$$
\begin{gathered}
f_{C}^{n}(z)-z=0, \\
f_{C}^{n^{\prime}}(z)-1=0 .
\end{gathered}
$$

The model local unfolding of such a point, for $n=1$, is

$$
f_{C}: z \mapsto C+z+z^{2} .
$$

This model is exactly the same as the quadratic family $z^{2}+C$, after translating the cardioid cusp point to the origin in both the $z$ and $C$ variables. The set of 
parameter values where this map has a fixed point with a derivative of norm one forms a cardioid curve in the $C$ plane, a translate of the cardioid in Fig. 2. The fact that the full curve is a cardioid is not as important to the local analysis as is the fact that there is a cusp at the origin. For $C$ values outside the cardioid, the corresponding maps have two repelling fixed points; for $C$ values inside the cardioid, the corresponding maps have an attracting and a repelling fixed point. On the cardioid curve, the maps have one linearly neutral and one repelling fixed point. The two fixed points coalesce, resulting in a single linearly neutral fixed point at $z=0$ as $C$ arrives at the cusp. These properties are well-known and easily verified by solving Eq. (6) for the model map in (5). Note in particular that saddle-node points are determined in the two-complex-dimensional $(z, C)$ space by two complex equations. We therefore expect solutions to be isolated points. This is in contrast with the real case, discussed in subsection 1.2.1 below, where the saddle-node set generically is a curve.

\subsubsection{Period-doubling points}

A period-doubling point is one such as the contact point between the main cardioid and its period-two bulb. It turns out that in the complex case such points are treated exactly as any other bulb-to-bulb contact point. These contact points are treated in the next subsection as resonant Hopf points. We include the separate subsection here only to emphasize that the period doubling case will be distinct for $\alpha \neq 0$. As with the saddle-nodes, period-doublings are generically points in the complex phase $\times$ parameter space $\mathcal{C} \times \mathcal{C}$, but curves in the real space $\mathcal{R}^{2} \times \mathcal{R}^{2}$.

\subsubsection{Hopf curves, resonant Hopf points, and bulb tangencies}

A fixed or periodic point changes stability as its derivative crosses the unit circle. Thus at bifurcation, we have a derivative of $e^{2 \pi i \omega}, \omega \in(0,1)$. (The $\omega=0$ case was already treated in subsection 1.1.1 above.) We are labelling such a bifurcation a Hopf bifurcation, unless $\omega=\frac{1}{2}$, when it is labelled a perioddoubling bifurcation. The required equations are

$$
\begin{gathered}
f_{C}^{n}(z)-z=0, \\
\left|f_{C}^{n^{\prime}}(z)\right|=1, \\
f_{C}^{n^{\prime}}(z) \neq 1 .
\end{gathered}
$$

Generically, bulb tangencies occur at every rational value of $\omega$. The model local unfolding for a fixed point with neutral rational derivative, which we call a $p / q$ resonant Hopf point, is

$$
f_{C}: z \mapsto e^{2 \pi i p / q}\left(z+C z+z^{q+1}\right)
$$

This model agrees, after standard changes of coordinates, up through degree $2 q$, with a generic $p / q$ resonant Hopf bifurcation. The set of parameter values 
where the map $f_{C}$ has a fixed point with a derivative of norm one forms a circle in the $C$ plane: center at -1 , radius of one. This is the parent bulb. The set of parameter values for which the maps have period- $q$ orbits with derivative of norm one forms a circle with center $1 / q$ and radius $1 / q$. This is the $p / q$ child bulb. Thus, locally, the two circles are tangent at the origin. There is a single fixed point and a single period- $q$ orbit for all parameter values except $C=0$. Inside the larger circle, the fixed point is attracting; it is neutral on the boundary and repelling outside. Similarly, the period- $q$ orbit is attracting inside its circle. The period- $q$ orbit collapses onto the fixed point $z=0$ as $C$ approaches zero. These facts can be verified directly from Eqs. (6) and (7). The only computational trick is to use the symmetry of the model family in order to solve for the period- $q$ points ( $n$ still is one) via $f_{C}(z)=e^{2 \pi i p / q} z$ instead of $f_{C}^{q}(z)=z$.

The collective result along a single Hopf curve is schematically depicted in Fig. 3a. Figure labels are more fully explained at the beginning of Sec. 2. Note that if one passes from a bulb to its $p / q$ child via a path nearby, but not directly through the contact point, the net result is the same, but the fixed point loses its stability before the period- $q$ point gains its stability. Of course, as one passes through the region exterior to both the parent bulb and the $p / q$ child bulb, the parameter value will typically pass through a multitude of other bulbs. This illuminates both the advantage and disadvantage of considering only a single parent-child pair at a time.

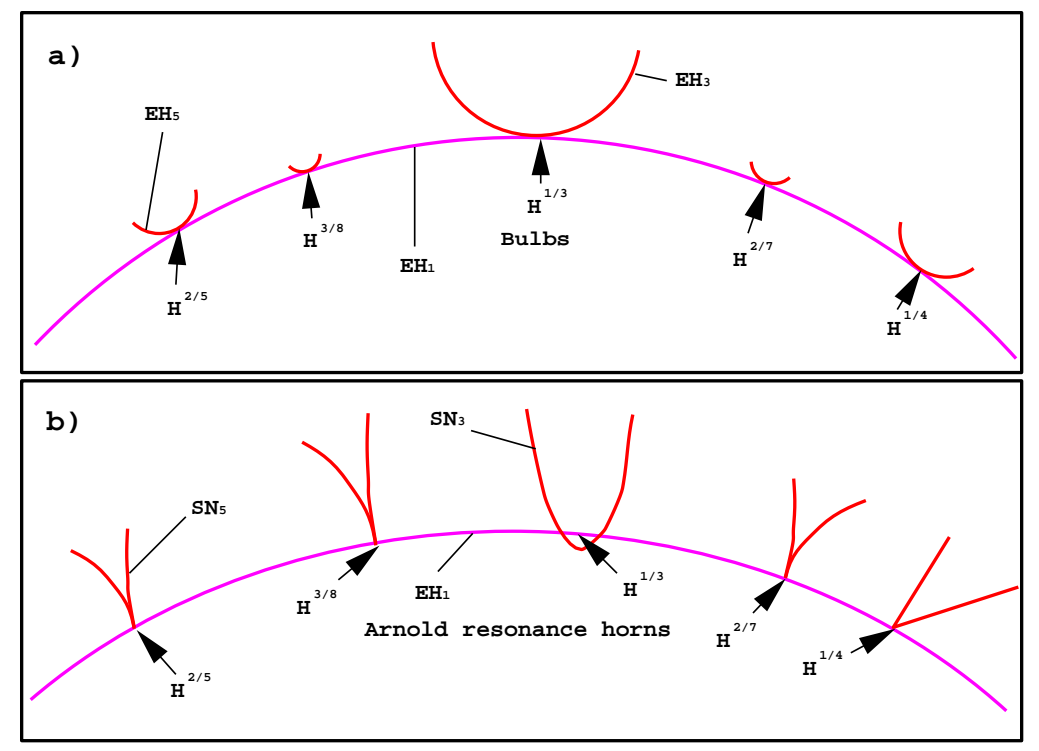

Figure 3: Generic Hopf bifurcation phenomena a)Bulb tangencies in the complex setting b) Arnold tongues in the real setting. 


\subsection{Maps of $\mathcal{R}^{2}$ : saddle-node, period-doubling, and Hopf bifurcations}

We will now recall some of the basic bifurcation facts for two-parameter families of maps of the plane.

Consider the two-parameter family of maps of the plane given by:

$$
(x, y) \mapsto R_{C}(x, y)
$$

where the phase variables $(x, y) \in \mathcal{R}^{2}$ and the parameter $C \in \mathcal{R}^{2}$. A bifurcation analyst trained to study real maps of the plane will analyze a map by first locating fixed points and determining their stabilities. In a two-parameter family of maps, the codimension-one bifurcation curves in the parameter plane, where changes in number or stability of the fixed points occur, will be then located. Both changes require a fixed point with at least one eigenvalue on the unit circle. These split into three generic cases: eigenvalue one (birth/death of a pair via saddle-node bifurcation), eigenvalue negative one (stability change via period-doubling bifurcation), and complex conjugate eigenvalues on the unit circle (stability change via Hopf bifurcation). After the local fixed-point bifurcations, one locates the same three local codimension-one bifurcations for period-two orbits, then period-three orbits, and so on. See Guckenheimer and Holmes [1983], Arnold [1983], and Ruelle [1989], for example. (These curves typically intersect at codimension-two points.)

We are interested in the loci of bifurcation points in the Cartesian product of the phase and parameter spaces, and in the projection of these loci to the parameter space. For all three loci, the first vector equation below is equivalent to two scalar equations and requires $(x, y)$ to be a period- $n$ point of $R_{C}$. The other equations are eigenvalue conditions. They are arrived at by recalling that an eigenvalue $\lambda$ of a $2 \times 2$ matrix $A$ satisfy $\lambda^{2}-\operatorname{tr}(A) \lambda+\operatorname{det}(A)=0 . D R_{C}^{n}$ is the two-by-two Jacobian derivative matrix of $R_{C}^{n}$, the $n^{\text {th }}$ iterate of $R_{C}$.

\subsubsection{Saddle-node curves}

The loci of period- $n$ saddle-nodes for Eq. (8) is determined by the following formulas.

$$
\begin{array}{cc}
R_{C}^{n}(x, y)-(x, y) & =0 \\
1-\operatorname{tr}\left(D R_{C}^{n}(x, y)\right)+\operatorname{det}\left(D R_{C}^{n}(x, y)\right) & =0
\end{array}
$$

The second equation requires an eigenvalue of one. This locus is generically a collection of curves in the four-real-dimensional phase $\times$ parameter space.

The model of such a bifurcation is

$$
\left(\begin{array}{l}
x \\
y
\end{array}\right)=\left(\begin{array}{c}
C_{1}-x^{2} \\
y / 2
\end{array}\right)
$$


The bifurcation set is $\left\{\left(x, y, C_{1}, C_{2}\right): x=y=C_{1}=0\right\}$. For negative $C_{1}$ values there are no fixed points; for positive $C_{1}$ values, there are two fixed points, one attracting node and one saddle. The two fixed points approach each other as $C_{1}$ approaches zero from above.

\subsubsection{Period-doubling curves}

The loci of the period- $n$ period-doubling points is determined by

$$
\begin{array}{cc}
R_{C}^{n}(x, y)-(x, y) & =0 \\
1+\operatorname{tr}\left(D R_{C}^{n}(x, y)\right)+\operatorname{det}\left(D R_{C}^{n}(x, y)\right) & =0
\end{array}
$$

The second equation in $\left(P D_{n}\right)$ requires an eigenvalue of negative one. Like the saddle-node locus, the period-doubling locus is generically a collection of curves.

The model of such a bifurcation is

$$
\left(\begin{array}{l}
x \\
y
\end{array}\right)=\left(\begin{array}{c}
-\left(C_{1}+1\right) x+x^{3} \\
y / 2
\end{array}\right) .
$$

The bifurcation set is $\left\{\left(x, y, C_{1}, C_{2}\right): x=y=C_{1}=0\right\}$. For negative $C_{1}$ values there is an attracting fixed point but no period-two orbit; for positive $C_{1}$ values, the fixed point is unstable and there is a stable period-two orbit. The period-two orbit collapses on the fixed point as $C_{1}$ approaches zero from above.

\subsubsection{Hopf curves, resonant Hopf points, and Arnold tongues}

A fixed or periodic point changes stability as its eigenvalues cross the unit circle. Avoiding the eigenvalues +1 and -1 , which correspond respectively to saddlenode and period-doubling bifurcations, this requires eigenvalues at bifurcation of $e^{ \pm 2 \pi i \omega}, \omega \in(0,0.5)$. Such a bifurcation is called a Hopf bifurcation. The required equations for a period- $n$ Hopf point are

$$
\left\{\begin{array}{c}
R_{C}^{n}(x, y)=(x, y)=0 \\
\operatorname{det}\left(D R_{C}^{n}(x, y)\right)-1=0
\end{array}\right\}\left(E H_{n}\right)
$$

The second equation of $\left(E H_{n}\right)$ requires the product of the eigenvalues to be 1 ; the inequality in $\left(H_{n}\right)$, along with the product of the eigenvalues being one, requires the eigenvalues to be complex conjugates on the unit circle. The notation $\left(E H_{n}\right)$ is used to stand for the Extended Hopf locus. The extended Hopf locus is a collection of curves which includes Hopf points as well as saddle points with real eigenvalues, such as 2 and $1 / 2$.

Generically (assuming $n=1$ for simplicity), for parameter values on one side of the Hopf bifurcation curve and away from $\omega=p / q$ with $q=1,2,3,4$, the corresponding maps have an invariant circle. This circle shrinks down onto the fixed point as the parameters approach the Hopf curve. 
The Hopf bifurcation for maps of $\mathcal{R}^{2}$ is much more subtle than the saddlenode or period-doubling bifurcations. This is because on the side of the Hopf bifurcation curve where the invariant circle exists, the parameter plane must be further divided in order to account for the births and deaths of periodic orbits of different periods that live on the invariant circle. Now classic results of Arnold [1983] and Takens [1974] guarantee that in a generic two-parameter family of maps of the plane (certainly not the case for a complex analytic family) the parameter space on the side of the Hopf curve with the invariant circles has a horn-shaped resonance region emanating from each point on the Hopf curve corresponding to a linearly neutral fixed point with rational eigenvalues. More precisely, the fixed point has eigenvalues $e^{ \pm 2 \pi i \omega}$ with $\omega=p / q$ and $q \geq 5$.

The model local unfolding for fixed points with neutral rational derivatives, which are called resonant Hopf points, is most conveniently presented in $(z, \bar{z})$ coordinates as:

$$
R_{C}: z \mapsto e^{2 \pi i p / q}\left(z+C z+A z^{2} \bar{z}+B \bar{z}^{q-1}\right) .
$$

The complex parameter $C$ is the unfolding parameter; $A$ and $B$ are fixed complex constants; $q \geq 3$. This map has a neutral fixed point for $C$ values on the circle with center at -1 and radius 1 . Inside the circle, the corresponding maps have an attracting fixed point; outside the fixed point is repelling. The side with the invariant circle is the side to which $-A$ points from the origin.

For $q \geq 5$, a resonance tongue, with tip at the origin, emanates from the origin in the direction of $-A$. The tip of the $p / q$ tongue is called a $p / q$ resonant Hopf bifurcation point. Inside a $p / q$ resonance tongue, the corresponding map has a pair of periodic orbits of period $q$ and rotation number $p / q$ when restricted to the invariant circle. Restricted to the circle, one of these orbits is attracting and one is repelling. The two side boundaries of a $p / q$ resonance tongue are period- $q$ saddle-node bifurcation curves (except where they meet at the resonant Hopf bifurcation point at the tip of the tongue).

When $q=3$, a so-called strong resonance case, a similar resonance tongue exists, but it does not have a tip on the Hopf curve. Instead, it swings "around" the parameter value of the $1 / 3$ Hopf point. When $q=4$, depending on the relative sizes of two coefficients in the normal form, the bifurcation diagram can resemble that of either $q=3$ or of $q \geq 5$. See Fig. 3b for a schematic depiction of the Hopf bifurcation diagram. In Fig. $3 \mathrm{~b}$ we arbitrarily assume the period-4 tongue resembles the $q \geq 5$ tongues.

For further insight into the resonant Hopf bifurcations, see McGehee and Peckham [1994] for a geometric presentation of the period- $q$ surface which exists in the neighborhood of a $p / q$ resonant Hopf point and period- $q$ saddle-node curves which run along the surface.

As with the saddle-node and period-doubling bifurcations, Hopf bifurcations of period- $n$ orbits can occur as well. They require the $n t h$ iterate of the map to have complex conjugate eigenvalues on the unit circle. 


\subsection{Complex vs. Real}

Although any one-complex-parameter family of complex-analytic maps of the complex plane can be thought of as a two-real-parameter family of maps of the real plane, it is worth emphasizing some of the major differences between real families which come from complex families and generic two-real-parameter families of maps of the plane.

\subsubsection{No births/deaths of orbits vs. births/deaths}

Since the complex numbers are a complete field, the number, counting multiplicity, of local roots of $f_{C}^{n}(z)-z$ cannot change with the parameter $C$. Thus, the only way for the number of roots to change is to have roots coalesce. This is what happens at the cusp of $\mathcal{M}$ and at the contact points between bulbs, as described in subsection 1.1 above. Note that for any family of constant degree polynomials, or of constant degree rational maps of the Riemann sphere, the global number of solutions of $f_{C}^{n}(z)-z$ also remains constant.

This local persistence of orbits is drastically different from what happens in generic real maps of $\mathcal{R}^{2}$. The saddle-node, period-doubling, and Hopf bifurcations all involve the births or deaths of new orbits.

\subsubsection{No saddles vs. saddles}

Any complex analytic map of the complex plane $z \mapsto f(z)$ can be viewed as a map from $\mathcal{R}^{2} \rightarrow \mathcal{R}^{2}$ via $(x, y) \mapsto(\operatorname{Re}(f(x+i y), \operatorname{Im}(f(x+i y))$. Note that the linear part of a complex map, $z \mapsto f^{\prime}(z) z$ then becomes

$$
\left(\begin{array}{l}
x \\
y
\end{array}\right) \mapsto\left(\begin{array}{cc}
\gamma & -\beta \\
\beta & \gamma
\end{array}\right)\left(\begin{array}{l}
x \\
y
\end{array}\right)
$$

where $f^{\prime}(z)=\gamma+i \beta$.

When viewed as a map of $\mathcal{R}^{2}$, this requires a complex analytic map with a real derivative to have a scalar multiple of the identity as its linear part. This is a nongeneric occurrence for two-parameter families of maps of $\mathcal{R}^{2}$. Also, it precludes having saddle points. Although we do not treat the subject much in this paper, this also has major consequences for the study of the dynamics of maps in the two settings. The stable and unstable manifolds of saddles often obviate the dynamics of a map of the plane, but complex analytic maps have no saddles.

The existence of saddles is related to subsection 1.3.4 as well.

\subsubsection{Bulb boundaries vs. Hopf curves}

We have already alluded to this earlier in the paper, but the relationship between the derivative of a complex map and the eigenvalues of the induced map of $\mathcal{R}^{2}$ 
makes it clearer why bulb boundaries would be considered as Hopf bifurcation curves by a "real" bifurcation analyst: a derivative of $e^{i \omega}$ for the complex map implies eigenvalues of $e^{ \pm i \omega}$ for the real map.

\subsubsection{Extended Hopf but not Hopf locus. Points vs. curves}

With our definition of the Hopf and Extended Hopf loci in subsection 1.2.3, the complex quadratic family would have only two points in the fixed point Extended Hopf locus that are not Hopf points: the cusp of the main cardioid and the period-doubling point. The cusp point has both eigenvalues at one; the period-doubling point has both eigenvalues at negative one. In the real setting, two-real-parameter unfoldings of points with double one eigenvalues (TakensBogdanov points [Bogdanov 1976],[Takens 1974]) result in a curve of Hopf points which terminates at the bifurcation point and continues as an Extended Hopf curve. The same is true for unfoldings of points with double negative one eigenvalues [Arnold 1983, Takens 1974]. Note that in the real case, the linear part of a double one eigenvalue point is generically a single Jordan block, while the linear part of the map induced from a complex map with eigenvalue one is the identity. A similar statement is true for the double negative one points.

\subsubsection{Bulb tangencies vs. Arnold tongues}

Compare again the Hopf bifurcation curves depicted in Fig. 3. The fixed point behavior is the same for each: there is a change from attracting to repelling as we cross the Hopf bifurcation curve. (This is also consistent with the fact that Eqs. (7) and (9) have the same linear part.) The $p / q$ bulbs in Fig. 3a and the $p / q$ tongues in Fig. 3b share the property that, inside each, there exists an attracting period- $q$ orbit while outside each, there is does not. The boundary of a $p / q$ bulb, however, is a Hopf curve, while the boundary of a tongue is a saddle-node curve. An extra pair of period- $q$ orbits, born in the saddle-node bifurcation, exists inside the tongue; there is only one period- $q$ orbit which is present in the deleted neighborhood of the bulb tangency.

\subsection{Intuition for blowup}

It is relatively obvious that bifurcation points for the complex analytic families should evolve into bifurcation regions after perturbation: period-doubling and saddle-node loci, including the sides of Arnold tongues, are generically curves in the real setting. How and under what conditions these blowups occur, however, is not so obvious. The rest of the paper addresses these issues. In Sec. 2, we describe for the model family partial bifurcation diagrams in the $C$ plane

for three values of $\alpha$. In Sec. 3, we prove theorems about the evolution of bifurcation points into regions after perturbation. 


\section{Numerical Investigation}

The numerical investigation in this paper was done on the model family defined in (1). We recall it here:

$$
z \mapsto F_{(C, \alpha)}(z, \bar{z})=z^{2}+C+\alpha \bar{z} .
$$

The cartesian coordinate version is in Eq. (2). We have numerically computed many bifurcation sets in the $C$ parameter plane for the following fixed values of $\alpha: 0,-0.1,+0.1$. All bifurcation curves in Figs. 2,4 , and 5 were computed using continuation software developed by the author [Peckham 1985-1997]. These are necessarily incomplete diagrams since each diagram has an infinity of bifurcation curves associated with it. Because all bifurcation diagrams are symmetric about the $\operatorname{Re}\{C\}$ axis $\left(F_{(C, \alpha)}\right.$ is conjugate to $F_{(\bar{C}, \alpha)}$ via $\left.z \mapsto \bar{z}\right)$, we have avoided computing most curves which lie completely below this axis.

We also indicate partial phase portraits in several regions through which the main cardioid passes. Paths through these regions are chosen so that the phase portraits at the ends of the paths are the same for $\alpha=0$ and $\alpha \neq 0$, but the intermediate portraits are more interesting when $\alpha \neq 0$. The phase portraits are partial because we consider only the existence and stability of the orbits corresponding to the parent and its $p / q$ child. Furthermore, we consider only one child at a time, even though the dynamics for the parameter regions we describe include higher period orbits as well.

Compare Figs. 8-11 in Bielefeld et. al. [1993] with our Figs. 4b, 5, 6; there are some common features.

Figure labels. In Figs. 4 and 5, saddle-node curves are colored green, period doubling curves are colored blue, and extended Hopf curves are colored red or magenta: red for the main cardioid region and its grandchildren, magenta for its children. In all figures, the saddle-nodes, period doublings and extended Hopf curves are labelled $S N_{n}, P D_{n}$, and $E H_{n}$, respectively, with the subscript denoting the period of the lowest period orbit involved in the bifurcation. Lines with an arrowhead point to codimension-two bifurcation points, labelled $C_{n}$ for a cusp point (saddle-node with a higher order degeneracy), $B_{n}$ for TakensBogdanov point (double one eigenvalue point), $D_{n}$ for a degenerate perioddoubling point (period-doubling point with a higher order degeneracy), $N_{n}$ for a double negative one eigenvalue point, and $H^{\frac{p}{q}}$ for a $p / q$ resonant Hopf point. See the following references for descriptions of these codimension-two points: Arnold [1983], Bogdanov [1976], Guckenheimer and Holmes [1983], Peckham and Kevrekidis [1991], Ruelle [1989], Takens [1974].

In Figs. 6-8, circles are fixed points; squares are period-2 points in Fig. 7, period-5 points in Fig. 8. Open symbols indicate repelling, plusses or x's inside other symbols indicate saddles, and filled symbols indicate attracting orbits. 


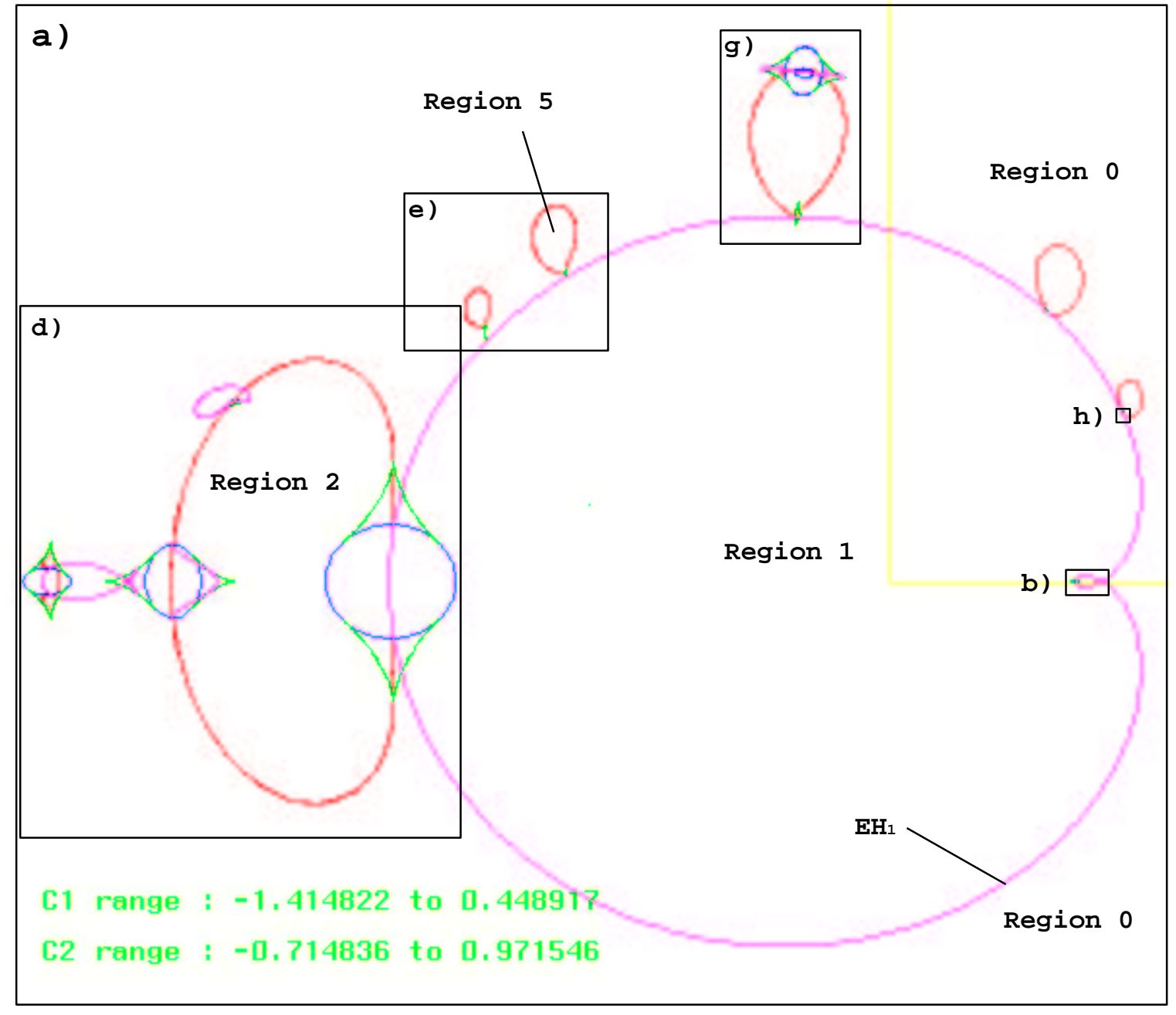

Figure 4: Numerically computed $C$ plane bifurcation diagrams for $\alpha=-0.1$. a) all computed curves, b,d,e,g,h) enlargements of (a), c) enlargement of (b), f) enlargement of $(\mathrm{e})$. 

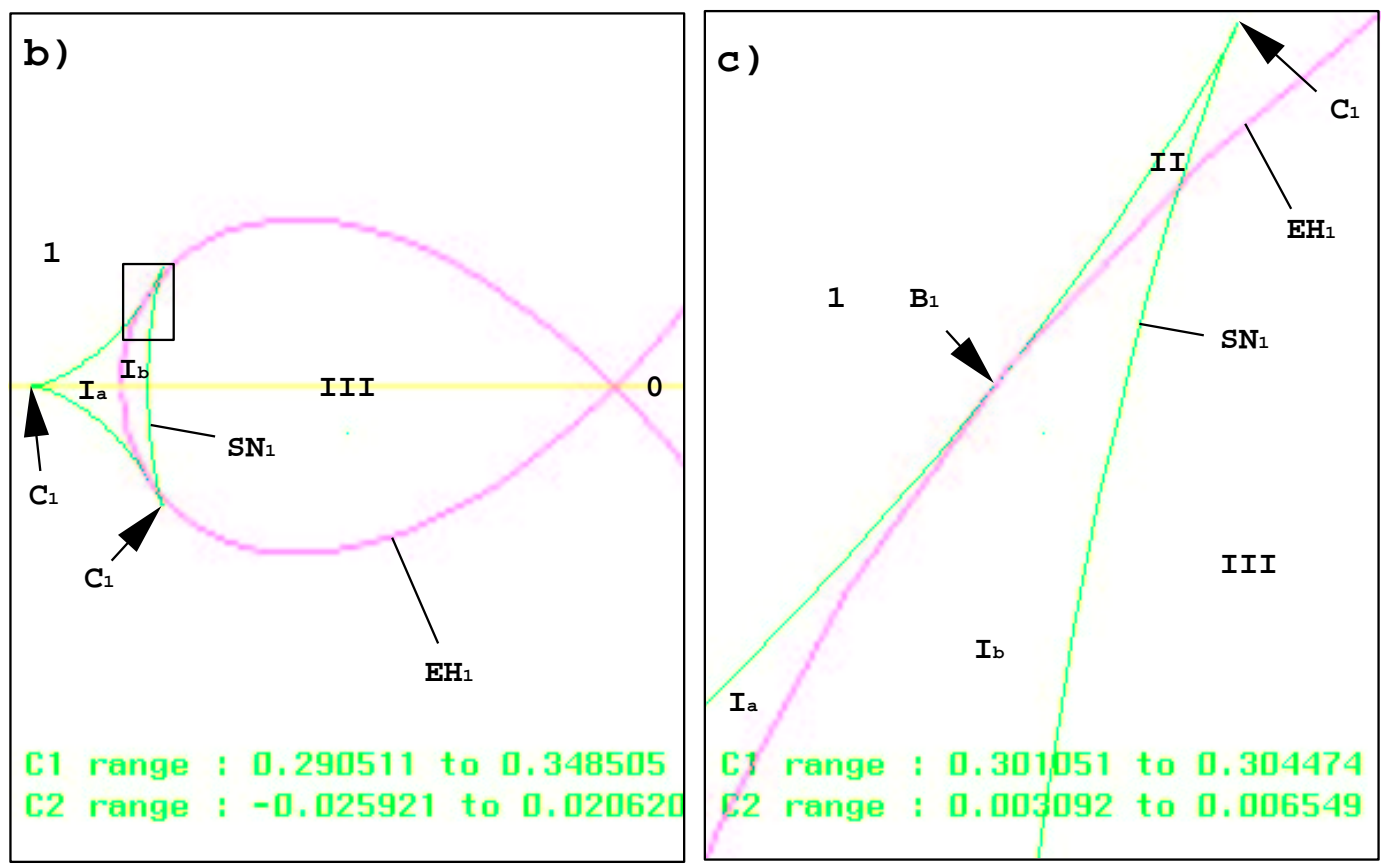

Figure 4 continued.

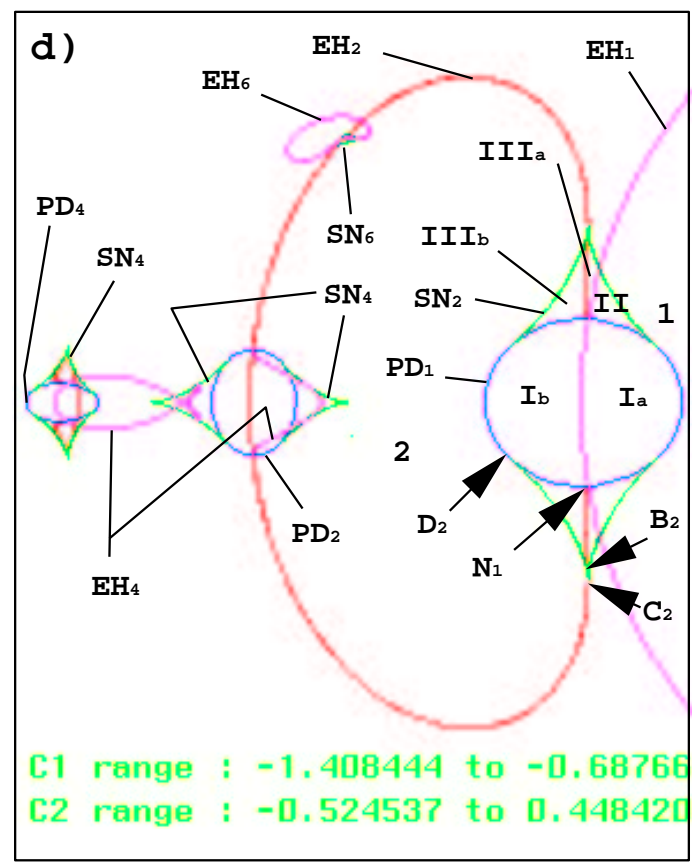

Figure 4: Numerically computed $C$ plane bifurcation diagrams for $\alpha=-0.1$. a) all computed curves, b,d,e,g,h) enlargements of (a), c) enlargement of (b), f) enlargement of (e). 

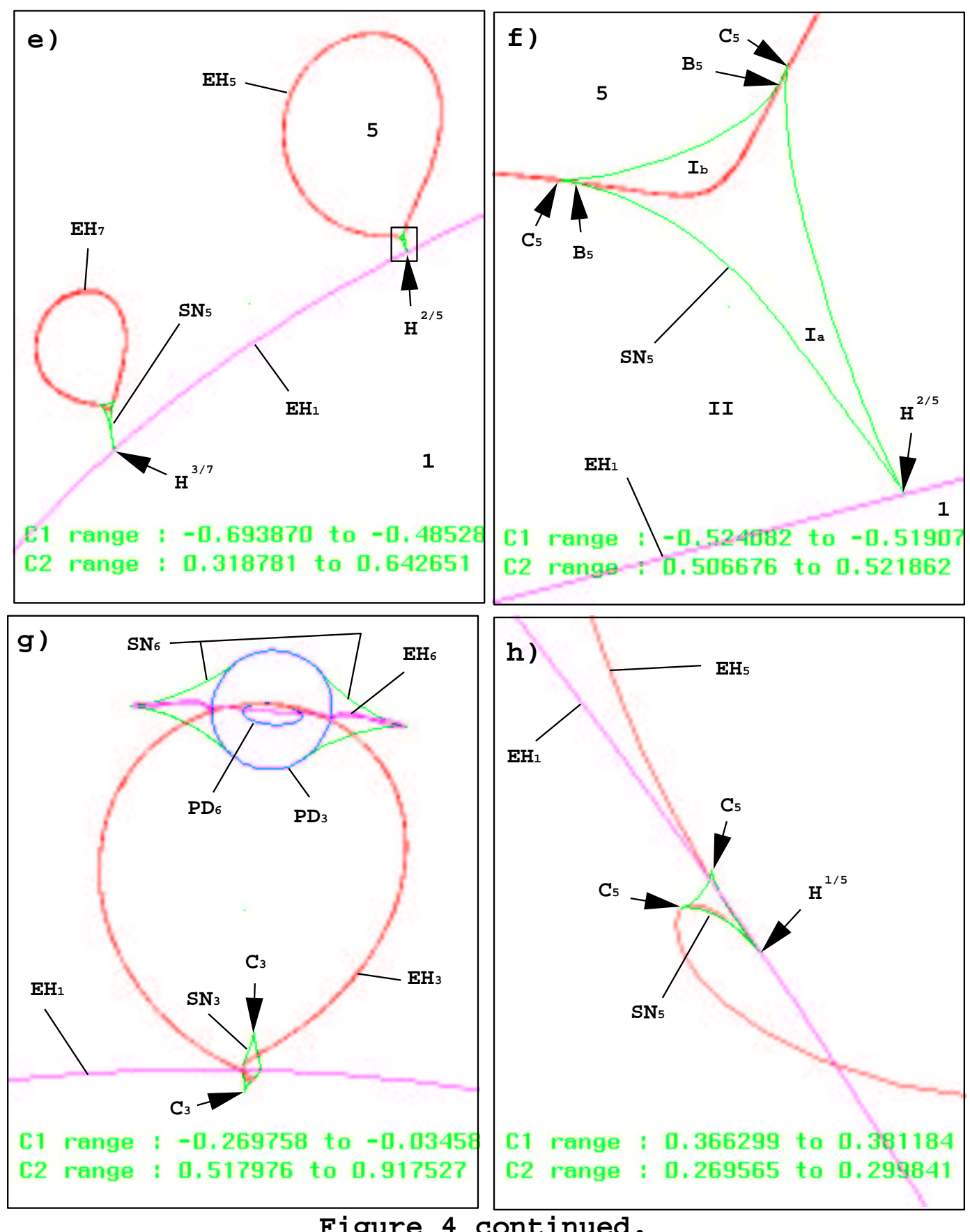

Figure 4: Numerically computed $C$ plane bifurcation diagrams for $\alpha=-0.1$. a) all computed curves, b,d,e,g,h) enlargements of (a), c) enlargement of (b), f) enlargement of (e). 


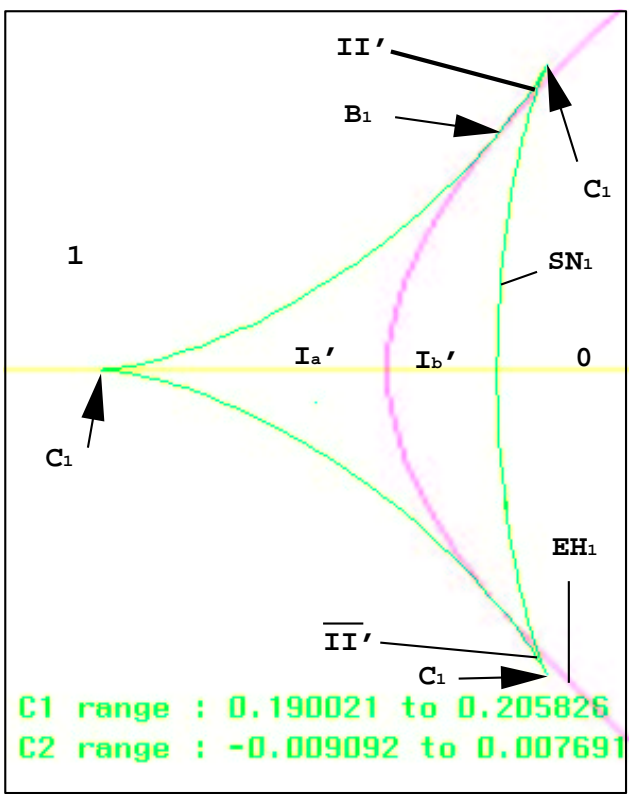

Figure 5: Enlargement of parameter plane near the cardioid cusp for $\alpha=+0.1$.

\section{$2.1 \quad \alpha=0$ : Figure 2}

We have computed several bulb boundaries. These are contained in the boundary of the Mandelbrot set. The local dynamics is consistent with that described in subsection 1.1.

Cardioid cusp point. As one crosses from region 1 inside the main cardioid to region 0 outside the main cardioid through the cusp $H^{\frac{0}{1}}$ ( $C$ passing through $1 / 4$ on the real axis), two real fixed points, one attracting and one repelling, come together. As $C$ grows to be greater than $1 / 4$, the fixed points separate as complex conjugates, both repelling. This is as described in subsection 1.1.1. See the region 1 and region 0 phase portraits in Fig. 6 .

Period-doubling point. As the period-doubling point $H^{\frac{1}{2}}$ is approached from inside the main cardioid (region 1), a repelling period-two orbit collapses on the attracting fixed point. Inside the period-two bulb (region 2), the periodtwo orbit separates from the fixed point. It is now stable and the fixed point is unstable. The repelling fixed point on the right plays no role in the transition. See the region 1 and 2 phase portraits in Fig. 7. Except for the orientation of the bulbs, this is as described in subsection 1.1.3.

Resonant Hopf points. Some discussion of this case was already given in subsection 1.1.3. The bulb-to-bulb transitions are all similar to the transition through the period-doubling point. For example, as $H^{\frac{2}{5}}$ is approached from 


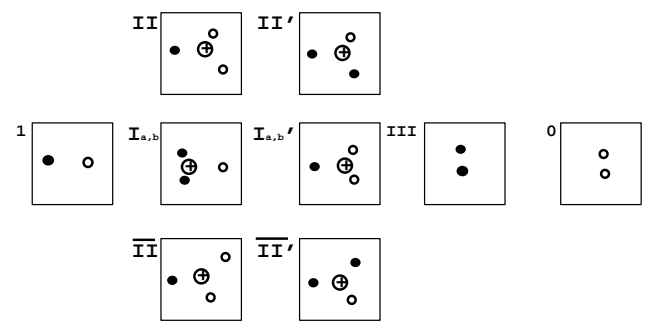

Figure 6: Fixed-point phase portraits near the cardioid cusp.

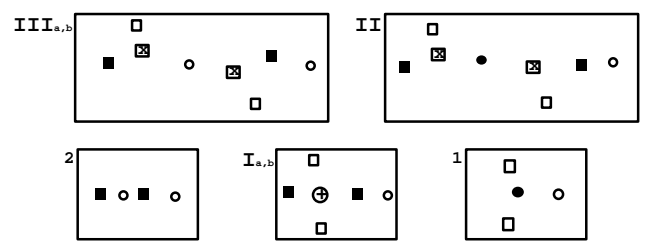

Figure 7: Fixed and period-two point phase portraits near the period doubling point.

inside the main cardioid (region 1), a repelling period-5 orbit collapses on the fixed point. Inside the $2 / 5$ bulb (region 5 ), the period-5 orbit separates from the fixed point, stability having been transferred from the fixed point to the period-5 orbit. See the region 1 and 5 phase portraits in Fig. 8. Note that there are a repelling fixed point and several other period-5 orbits that play no role in the local bifurcation. These orbits are not represented in the Fig. 8 phase portraits.

\section{$2.2 \alpha=-0.1$ : Figure 4}

For $\alpha \neq 0$, the resulting two-parameter family of maps is no longer complex analytic, and we expect that it is a generic two-real-parameter family of maps of the real plane. Thus we no longer expect to see bifurcations which are of codimension above two (codimension defined as in the real setting). In particular, the cusp point of the main cardioid, and the period-doubling point of the main cardioid are now nongeneric. A partial picture is illustrated in Fig. 4a. Rectangles are drawn around regions whose enlargements are shown in other parts of the Fig. 4.

Cardioid cusp point blowup. Look first at the region near where the cusp of the main cardioid was for $\alpha=0$. Enlargements are shown in Figs. $4 \mathrm{~b}$ and 4c, with corresponding phase portraits in Fig. 6. The cusp on the Extended Hopf curve has simultaneously evolved into a smooth loop and grown a triangular curve of saddle-node points. There are two points in the four-dimensional $(z, C)$ space where the Extended Hopf and saddle-node curves intersect. Their 

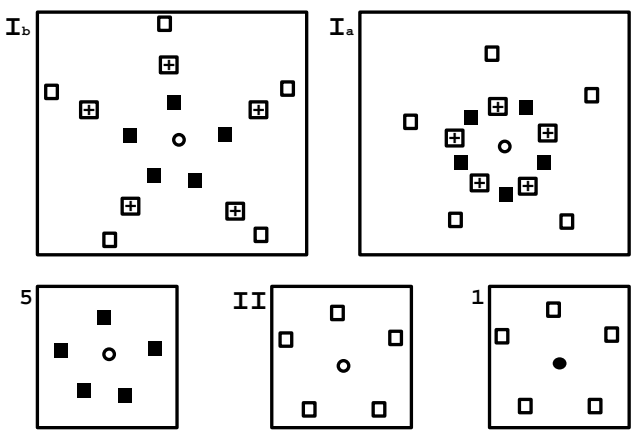

Figure 8: Fixed and period-five point phase portraits near period-5 2/5 resonant Hopf point.

projections to the $C$ parameter plane are the indicated Takens-Bogdanov points. The top one is labelled $B_{1}$ in Fig. 4c. Such points have both eigenvalues equal to one but a one-dimensional associated eigenspace. In between the two Takens-Bogdanov points, the Extended Hopf curve is not a true Hopf curve: the eigenvalues of the corresponding fixed point are real. Thus, there is no local bifurcation between regions $I_{a}$ and $I_{b}$. Recall that we are ignoring global bifurcations in this study, although such bifurcations certainly do play a role. See Peckham [1988] or Vance and Ross [1989] for bifurcation studies which do include global bifurcations. Although those two bifurcation studies are of forced oscillator systems, they have features similar to this study because both settings include generic two-real-parameter Hopf bifurcation phenomena.

Consider a path in Fig. 4b from region 1 to $I_{a}$ to $I_{b}$ to $I I I$ to 0 . For $C$ parameter values in region 1 and region 0 , the fixed-point phase portraits are the same as for the $\alpha=0$ case: one attracting and one repelling fixed point exist in region 1, while two repelling fixed points exist in region 0 . But the path from region 1 to region 0 is now much more complicated. As one enters region $I_{a}$ from region 1, a new pair of fixed points is born in a saddle-node bifurcation (a pitchfork bifurcation if one passes through the cusp $C_{1}$ on the $\operatorname{Re}\{C\}$ axis); one new fixed point is a saddle and the other an attracting node. As one passes from region $I_{b}$ to region $I I I$, the saddle and the unique repelling fixed point coalesce and disappear, again at a saddle-node bifurcation. The two attracting fixed points that exist in region $I I I$ then change to repelling, simultaneously if we restrict $C$ to its real axis, leaving us with two repelling fixed points by the time we enter region 0 . The end result is that a fixed point has changed from attracting to repelling, just as happened as we passed from region 1 to region 0 in the $\alpha=0$ case.

Note that the cusps on the saddle nodes are necessary features: that is what enables the saddle to be born with one fixed point (an attracting one) and die with another (the repelling one). The saddle "changes partners" at the cusp 
point.

If one passes through region $I I$, shown in Fig. 4c, or $\overline{I I}$, its conjugate in the $C$ plane, the sequence indicated in Fig. 6 is slightly more complicated, but the net result of going from region 1 to region 0 is, of course, the same.

Period-doubling point blowup. See the parameter space enlargement in Fig. 4d and corresponding phase portraits in Fig. 7. The period-doubling point has evolved into a period-doubling circle, $P D_{1}$. The extended Hopf curve $E H_{1}$ passes through the circle, intersecting it in the two double-negative-one eigenvalue points, the lower one only labelled, as $N_{1}$. Remember the bifurcation diagram is symmetric about the $\operatorname{Re}\{C\}$ axis, so labels have been made either in the top or bottom half only. In between the two double-negative-one eigenvalue points, the extended Hopf curve corresponds to saddle points, so there is no local bifurcation between regions $I_{a}$ and $I_{b}$. A variety of scenarios is possible in going from region 1 to region 2. The most direct route is region 1 to $I_{a}$ to $I_{b}$ to 2 . It involves the birth of a single attracting period-two orbit in a period-doubling bifurcation (region 1 to region $I_{a}$ ), and a death of the repelling period-two orbit in a second period-doubling bifurcation (region $I_{b}$ to region 2). This pair of bifurcations changes the attracting fixed point first to a saddle and then to a repeller. Thus we end up in region 2 as we did for the $\alpha=0$ case, with an attracting period-2 orbit and two repelling fixed points. Other scenarios can be seen from the figures. If we enlarged the region of Fig. $4 \mathrm{~d}$ containing a period-two Takens-Bogdanov point $\left(B_{2}\right)$ and a period-two cusp point $\left(C_{2}\right)$, the diagram would resemble the period-one scenario in the Fig. 4c enlargement.

Resonant Hopf points: growth of Arnold tongues. Except for the period doubling bifurcation just described above, the description of the bifurcations which have evolved from the points which were tangencies between the main cardioid and some other bulb for the $\alpha=0$ case are all roughly similar.

First consider the bifurcations near the $2 / 5$ bulb: from region 1 to $I I$ to $I_{a}$ to $I_{b}$ to 5 , in Figs. 4e and 4f. This bulb was chosen because the 2/5 Arnold tongue conecting the main cardioid and the $2 / 5$ bulb is oriented in the simplest possible way. The accompanying phase portraits are in Fig. 8. The bifurcation diagram is similar in some ways to the diagram described above for the cardioid cups point blowup. There is a triangular region of saddle-node curves (the golf tee, according to Rick Moeckel) inside which there exists an "extra pair" of period-5 orbits. As one enters the triangular region from either side (and below $B_{5}$ on the left side), an extra saddle orbit and an extra attracting node orbit are born. When close enough to the resonant Hopf point $H^{\frac{2}{5}}$ tip of the tongue, the two new period- 5 orbits live on an invariant circle. This is suggested in phase portrait $I_{a}$. There is no change in stability between regions $I_{a}$ and $I_{b}$, but the invariant circle breaks and the orbits reallign themselves in preparation for the saddle-node bifurcation at the top of the sadd-node triangle. As one passes out the top of the triangular region (and to the left of $B_{5}$ near the right corner), the saddle period- 5 orbit dies with a repelling period- 5 orbit. In region 
5, the dynamics are similar to being in the period- 5 bulb of the Mandelbrot set: there is an attracting period- 5 orbit and all other periodic orbits are (appear to be) repelling. The main difference between this case and the saddle-node point blowup case is that the in the saddle-node point blowup, the three corners of the saddle-node triangle are all cusps $\left(C_{1}\right.$ in Fig. $\left.4 \mathrm{~b}\right)$, while in this case the corners are two cusps $\left(C_{5}\right)$ and one resonant Hopf bifurcation point $\left(H^{\frac{2}{5}}\right)$.

Now consider the enlargement of the region near the $1 / 3$ bulb shown in Fig. $4 \mathrm{~g}$. We do not describe the phase portraits since they can largely be inferred from the phase portraits we have already described. We do, however, make the following observations. The period-three saddle-node curve has two cusp points $C_{3}$, but does not contain the resonant Hopf point $H^{\frac{1}{3}}$. This is consistent with the generic Hopf bifurcation picture of figure 3b. Another interesting observation is the relative positions of the two period-doubling circles: $P D_{3}$ and $P D_{6}$. The period-six period-doubling circle has moved, as $\alpha$ has increased from zero, from a position necessarily above the period-three period-doubling circle to the position shown in Fig. 4g, completely inside the period-three period doubling circle. This has been accompanied by the flattening of the extended Hopf curve $E H_{6}$, which used to be the period-six bulb boundary. This suggests the formation of a nested sequence of period-doubling circles and possibly the formation of a period-doubling route to chaos.

Finally, consider the enlargement Fig. $4 \mathrm{~h}$ near the $1 / 5$ resonant Hopf point. The Arnold tongue is not so nicely oriented as is the $2 / 5$ tongue in Fig. 4f. The $1 / 5$ tongue suggests nearby Hopf bifurcations are subcritical. We include no more phase portraits for the $1 / 5$ region, but only point out that the bifurcation scenario there is more complicated than in the $2 / 5$ region.

\section{$2.3 \alpha=+0.1$ : Figure 5}

The description of the $\alpha=+0.1$ bifurcations is quite similar to that of the $\alpha=-0.1$ bifurcations. The scenario of the saddle-node point blowup is even simpler than for $\alpha=-0.1$ because the cusp perturbs to a smooth curve without a loop. An enlargement of this region in figure 5. This is the only part of the bifurcation diagram we show for $\alpha=+0.1$. Corresponding phase portraits are in figure 6 .

The scenario for the $\alpha=+0.1$ period-doubling point blowup is more complicated than for $\alpha=-0.1$. It is, however, like the scenario for $\alpha=-0.1$ perioddoubling point blowup of the period-doubling point between the $1 / 2$ bulb and its $1 / 2$ child. See Fig. 4 d again. Note in particular that the period-two extended Hopf curve, labelled $\mathrm{EH}_{4}$ has two components, and the two cusped saddle-node curves, $S_{4}$ are attatched on the left and right of the period-doubling circle $P D_{2}$ rather than above and below it, as the two saddle-node curves $S N_{2}$ are attatched to the period-doubling circle $P D_{1}$. For both $\alpha=-0.1$ and $\alpha=+0.1$ the period-doubling point blowups computed alternate between the simpler and more complicated types. 
The resonant Hopf point blowups are generally similar to the $\alpha=-0.1$ case. No new figures are included for $\alpha=+0.1$.

\subsection{Shrinking back to points}

It is useful to view again the bifurcation curves of Fig. 4a or any of its enlargements and imagine what happens as $\alpha$ is increased toward zero. The green saddle-node and blue period-doubling sets, and the enclosed regions and curves, must all shrink back to a point, leaving us with the Mandelbrot set boundary of Fig. 2.

\section{Theorems}

In this section we provide sufficient conditions for three types of bifurcation points in a complex analytic family to evolve into a bifurcation region after perturbation by a real function which is not complex analytic. The three types are the cardioid cusp point, the period-doubling point, and the resonant Hopf bifurcation point.

General setup. For all three theorems, the general form we consider has $F_{(\alpha, C)}: \mathcal{D} \rightarrow \mathcal{C}$, where $\mathcal{D}$ is a neighborhood of the origin in $\mathcal{R}^{2}$, or by the obvious equivalence, a neighborhood of the origin in $\mathcal{C} . F_{(\alpha, C)}$ is defined by

$$
F_{(\alpha, C)}(z, \bar{z})=f_{C}(z)+\alpha g_{\alpha}(z, \bar{z})
$$

where $z=x+i y=r e^{i \theta} \in \mathcal{C}, C=C_{1}+i C_{2} \in \mathcal{C}, \alpha \in \mathcal{R}$ and $\bar{z}=x-i y$ is the complex conjugate of $z$. We assume that $f_{C}(z)$ is a one-complex-parameter family of complex analytic maps defined on $\mathcal{D}$ and depending complex analytically on the parameter $C$. We also assume that $g_{\alpha}(z, \bar{z})$ is a $C^{\infty}$ map of $\mathcal{R}^{2}$ depending smoothly $\left(C^{\infty}\right)$ on the real parameter $\alpha$. Thus we can expand $f_{C}(z)=a_{0}(C)+a_{1}(C) z+a_{2}(C) z^{2}+a_{3}(C) z^{3}+\ldots$ and $g_{\alpha}(z, \bar{z})=g_{00}(\alpha)+$ $g_{10}(\alpha) z+g_{01}(\alpha) \bar{z}+g_{20}(\alpha) z^{2}+g_{11}(\alpha) z \bar{z}+g_{02}(\alpha) \bar{z}^{2}+\ldots$, where each $a_{k}(C)$ is complex analytic on some neighborhood of the origin, and each $g_{j k}(\alpha)$ is $C^{\infty}$ in some neighborhood of the origin. We will, by a slight abuse of notation, sometimes think of $z$ and $C$ as variables in $\mathcal{R}^{2}$ by identifying $z$ with $(x, y)$ and $C$ with $\left(C_{1}, C_{2}\right)$.

Remarks. All results are stated using the more computationally convenient " $(z, \bar{z})$ " coordinates. The "base" map for all three cases has a complex-codimensionone degeneracy associated with it (a fixed point with a certain neutral eigenvalue $a_{1}(0): f_{0}(z)=a_{1}(0) z+a_{2}(0) z^{2}+\ldots ; f_{C}$ is a generic one-complex-parameter complex analytic unfolding of the base map; $g_{\alpha}: \mathcal{R}^{2} \rightarrow \mathcal{R}^{2}$ is the real perturbing map, and $\alpha$ the perturbing parameter. Although $g_{\alpha}$ is assumed to be $C^{\infty}$, it 
really only needs to be $C^{k}$ with $k$ large enough so that $g_{\alpha}$ can be expanded in a power series in $z, \bar{z}$, and $\alpha$ up to the order indicated in the theorems. We consider the two-real-parameter bifurcation diagrams for $\alpha$ fixed. In particular, we discuss when a bifurcation point in the $C$ plane for $\alpha=0$ blows up into a bifurcation region for $\alpha$ nonzero. All results are local in the variables $(z, C, \alpha)$ in the five-real-dimensional space $\mathcal{C} \times \mathcal{C} \times \mathcal{R}$. The second and third theorems are stated assuming the expansions of the complex-analytic family $f_{C}(z)$ and the perturbing family $g(z, \bar{z})$ are in normal form.

Theorem 3.1 The cardioid cusp point blowup. Let $F_{(\alpha, C)}(z, \bar{z})$ be defined as above in Eq. (10). Assume the accompanying general setup and

1. Eigenvalue one condition for $f_{0}: a_{0}(0)=0, a_{1}(0)=1$

2. Higher order nondegeneracy condition for $f_{0}: a_{2}(0) \neq 0$

3. Unfolding nondegeneracy condition for $f_{C}: a_{0}^{\prime}(0) \neq 0$ (The prime indicates the complex derivative.)

4. Blowup nondegeneracy condition on $g_{\alpha}: g_{01}(0) \neq 0$.

Then, near $(0,0,0),\left\{(z, C, \alpha) \in \mathcal{R}^{2} \times \mathcal{R}^{2} \times \mathcal{R}: z\right.$ is a fixed point for $F_{(\alpha, C)}$ with eigenvalue 1$\}$ is a topological cone. Each constant $\alpha$ cross section is

- a nondegenerate topological circle if $\alpha \neq 0$. This circle also projects nontrivially (not as a point) the $C$ parameter plane.

- $a$ point if $\alpha=0$.

The sketch of the proof is as follows. We first make an $\alpha$ and $C$ dependent translation in $z$ to keep the coefficient of $z$ in our family at one, independent of the parameters $C$ or $\alpha$. This is what we consider our normal form for this bifurcation. Since it is not as well-known as the normal forms for the bifurcations of Theorems 2 and 3, we include it in Sec. 4 as the Cardioid cusp normal form lemma. Next we solve, using the implicit function theorem for $C$ as a function of $\alpha$ and $z$ to ensure that $z$ is a fixed point for $F_{(\alpha, C)}$. Then we substitute into the eigenvalue one equation and collect the lowest order terms in our small parameters, $\alpha$ and $r\left(z=r e^{i \theta}\right)$. The result is then obvious. Details are in Sec. 4.

Theorem 3.2 The period-doubling point blowup. Let $F_{(\alpha, C)}(z, \bar{z})$ be defined as above in Eq. (10). Assume the accompanying general setup and

1. Eigenvalue negative one condition for $f_{0}: a_{0}(0)=0$ and $a_{1}(0)=-1$

2. Normal form assumption through order three for $f_{C}$ and $g_{\alpha}$ : Coefficients $a_{0}(C), a_{2}(C), g_{00}(\alpha), g_{20}(\alpha), g_{11}(\alpha), g_{02}(\alpha)$, are identically zero (Any term with even total power-the sum of the powers of $z$ and $\bar{z}-$ can be eliminated by a near identity change of coordinates.) 
3. Higher order nondegeneracy condition for $f_{0}: a_{3}(0) \neq 0$

4. Unfolding nondegeneracy condition for $f_{C}: a_{1}^{\prime}(0) \neq 0$ (The prime indicates the complex derivative.)

5. Blowup nondegeneracy condition for $g_{\alpha}: g_{01}(0) \neq 0$.

Then, near $(0,0,0),\left\{(z, C, \alpha) \in \mathcal{R}^{2} \times \mathcal{R}^{2} \times \mathcal{R}: z\right.$ is a fixed point for $F_{(\alpha, C)}$ with eigenvalue -1$\}$ is a topological cone. Each constant $\alpha$ cross section is

- a nondegenerate topological circle if $\alpha \neq 0$. This circle also projects to a nondegenerate topological circle in the $C$ parameter plane.

- $a$ point if $\alpha=0$

The proof is much simpler than the saddle-node blowup proof because the unique fixed point is always at the origin in the normal form. Details are in Sec. 4.

It turns out that if $g_{01}(0) \neq 0$ then the radius of the circular cross sections is $O(\alpha)$. The theorem is true for a generic base map having a fixed point with derivative -1 by first converting to the normal form in the statement of the theorem.

Theorem 3.3 The bulb contact point blowup. Let $F_{(\alpha, C)}(z, \bar{z})$ be defined as above in Eq. (10). Assume the accompanying general setup and

1. $p / q$ resonant Hopf eigenvalue condition: $a_{0}(0)=0$ and $a_{1}(0)=e^{2 \pi i p / q}$

2. Normal form assumption through order $q+1: a_{0}(C)=0, a_{k}(C)=0$ for $k=2,3, \ldots, q, g_{i j}(\alpha)=0$ for $i, j$ nonnegative integers whose sum is less than $q+1$ and $i-j-1$ is not a multiple of $q$.

3. Higher order nondegeneracy condition for $f_{0}: a_{q+1}(0) \neq 0$

4. Unfolding nondegeneracy condition for $f_{C}: a_{1}^{\prime}(0) \neq 0$ (The prime indicates the complex derivative.)

5. Blowup nondegeneracy conditions for $g_{\alpha}: g_{21}(0) \neq 0$ and $g_{0, q-1}(0) \neq 0$.

Then, if $q \geq 5$ (to avoid "strong resonances"), near $(0,0,0)$, each constant $\alpha$ cross section of $\left\{(z, C, \alpha) \in \mathcal{R}^{2} \times \mathcal{R}^{2} \times \mathcal{R}: z\right.$ is a period-q saddle-node point for $\left.F_{(\alpha, C)}\right\}$ is

- a nontrivial set including at least $2 q$ curves which join at the origin if $\alpha \neq 0$. This set projects to a set which includes the sides of an Arnold resonance tongue in the $C$ parameter plane

- $a$ point if $\alpha=0$. 
The proof is a direct application of results of Arnold [1983]. The hedge is that these results give only part of the bifurcation picture. The full picture for nonzero $\alpha$, and including the strong resonance cases $q=4$ or 5 , based on numerical continuations, is conjectured below.

Conjecture 3.4 The bulb contact point blowup conjecture. Assume the hypotheses of the Bulb contact point blowup theorem. Then, there exists a neighborhood $\mathcal{N}$ of $(0,0,0)$ such that each nonempty, constant $\alpha$ cross section of $\mathcal{N} \cap\left\{(z, C, \alpha) \in \mathcal{R}^{2} \times \mathcal{R}^{2} \times \mathcal{R}: z\right.$ is a period-q saddle-node point for $\left.F_{(\alpha, C)}\right\}$ is

- for $q \geq 5$ :

- a flower with $q$ petals if $\alpha \neq 0$. This flower projects to a triangle with three cusps in the $C$ parameter plane. One cusp is the resonant $p / q$ Hopf point; the other two are cusps which are the standard saddlenode with a higher order degeneracy.

- a point if $\alpha=0$.

- $\operatorname{for} q=3$ :

- a topological circle if $\alpha \neq 0$. This circle projects to a topological circle with two cusps in the $C$ parameter plane. The missing cusp from the $q \geq 5$ case is the cusp and the resonant Hopf point. Instead, the saddle-node curve swings around the resonant Hopf point in a smooth curve.

- a point if $\alpha=0$.

- for $q=4$ : This case is like the $q \geq 5$ case if $\left|g_{21}(0)\right|>\left|g_{03}(0)\right|$ and like case $q=3$ if $\left|g_{21}(0)\right|<\left|g_{03}(0)\right|$.

See the numerically computed projections to the phase space in Fig. 9. The projections to the parameter space were already shown: $S N_{5}$ in Fig. $4 \mathrm{f}$ and $S N_{3}$ in $4 \mathrm{~g}$.

See McGehee and Peckham [1994] for additional discussion of the geometry of fixed-point and periodic point surfaces, the saddle-node curves on the periodic point surface, the intersection of the saddle-node curves at the resonant Hopf point, and the projection of all these sets from the phase $\times$ parameter space to the parameter space.

\section{Proofs}

Lemma 4.1 The Cardioid cusp normal form lemma. Let $F_{\beta}(z, \bar{z})$ be a $C^{\infty} k$-complex-parameter family of maps of $\mathcal{R}^{2}$. Expand $F$ as $F_{\beta}(z, \bar{z})=$ $F_{00}(\beta)+F_{10}(\beta) z+F_{01}(\beta) \bar{z}+F_{20}(\beta) z^{2}+F_{11}(\beta) z \bar{z}+F_{02}(\beta) \bar{z}^{2}+\ldots$. Assume 

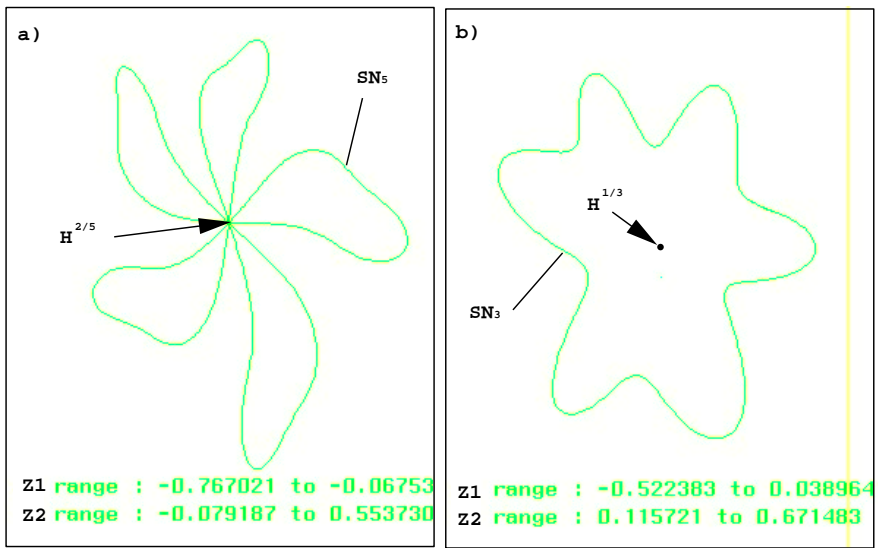

Figure 9: Numerically computed saddle-node loci for $\alpha=-0.1$, projected to the $z$ phase plane a) $2 / 5$ saddle-nodes, b) $1 / 3$ saddle-nodes.

$F_{0}$ is complex analytic, $F_{10}(0)=1$ and $F_{20}(0) \neq 0$. Then, there exists a $\beta$ dependent translation $\omega=z-T(\beta)$ such that, in the new variables, the resulting map has its linear coefficient always equal to one. That is, if $G$ is defined by $G_{\beta}(w, \bar{w})=F_{\alpha}(w+T(\beta), \bar{w}+\overline{T(\beta)})-T(\beta)$, and the expansion of $G$ is $G_{\beta}(w, \bar{w})=G_{00}(\beta)+G_{10}(\beta) w+G_{01}(\beta) \bar{w}+G_{20}(\beta) w^{2}+G_{11}(\beta) w \bar{w}+G_{02}(\beta) \bar{w}^{2}+$ $\ldots$, then $G_{10}(\beta)=1$.

Proof Define the translation by $\omega=z-T$. The definitions of $F$ and $G$ in the statement of the Lemma imply that $G$ has the form:

$$
\begin{gathered}
G_{\beta}(w, \bar{w})=F_{00}(\beta)+F_{10}(\beta) T+F_{01}(\beta) \bar{T}+O_{2}(T, \bar{T})-T \\
+w\left(F_{10}(\beta)+2 F_{20}(\beta) T+F_{11}(\beta) \bar{T}+O_{2}(T, \bar{T})\right) \\
+\bar{w}\left(F_{01}(\beta)+F_{11}(\beta) T+2 F_{02}(\beta) \bar{T}+O_{2}(T, \bar{T})\right) \\
+O_{2}(w, \bar{w}) .
\end{gathered}
$$

Thus $G_{10}(\beta)=F_{10}(\beta)+2 F_{20}(\beta) T+F_{11}(\beta) \bar{T}+O_{2}(T, \bar{T})$. Define $K(T, \bar{T}, \beta) \equiv$ $G_{10}(\beta)-1$. We want to show that there exists a complex-valued function $T(\beta)$ such that $K(T(\beta), \overline{T(\beta)}, \beta)=0$. This is equivalent to showing there exist two real valued functions $T_{1}(\beta)$ and $T_{2}(\beta)$ such that $\tilde{K}\left(T_{1}, T_{2}, \beta\right) \equiv K\left(T_{1}(\beta)+\right.$ $\left.i T_{2}(\beta), T_{1}(\beta)-i T_{2}(\beta), \beta\right)=0$. Since $F_{10}(0)=1$ by assumption, then $\tilde{K}(0,0,0)=$ 0 , and the functions $T_{1}(\beta)$ and $T_{2}(\beta)$ clearly exist by the implicit function theorem if

$$
\left.\operatorname{det}\left(\begin{array}{ll}
\frac{\partial \tilde{K}_{1}}{\partial T_{1}} & \frac{\partial \tilde{K}_{1}}{\partial T_{2}} \\
\frac{\partial \tilde{K}_{2}}{\partial T_{1}} & \frac{\partial \tilde{K}_{2}}{\partial T_{2}}
\end{array}\right)\right|_{\left(T_{1}, T_{2}, \beta\right)=(0,0,0)} \neq 0
$$


where $\tilde{K}_{1}=\operatorname{Re}(\tilde{K})$ and $\tilde{K}_{2}=\operatorname{Im}(\tilde{K})$ Straightforward differentiation shows this matrix is

$$
\left(\begin{array}{cc}
2 \operatorname{Re}\left(F_{20}(0)\right)+\operatorname{Re}\left(F_{11}(0)\right) & -2 \operatorname{Im}\left(F_{20}(0)\right)+\operatorname{Im}\left(F_{11}(0)\right) \\
2 \operatorname{Im}\left(F_{20}(0)\right)+\operatorname{Im}\left(F_{11}(0)\right) & 2 \operatorname{Re}\left(F_{20}(0)\right)-\operatorname{Re}\left(F_{11}(0)\right)
\end{array}\right) .
$$

Its determinant is $4\left|F_{20}(0)\right|^{2}-\left|F_{11}(0)\right|^{2}=4\left|F_{20}(0)\right|^{2} \neq 0$ since $F_{11}(0)=0(F$ is analytic at $\beta=0)$, and $F_{20}(0)$ was assumed to be nonzero.

Proof of the Cardioid cusp blowup theorem 3.1 We apply the lemma just proved to $F_{(\alpha, C)}$, with $\beta=(\alpha, C)$. We can think of $\alpha$ as complex for the application of the lemma, even though we are only interested in $\alpha$ real. Thus, we can assume that $a_{1}(C)+\alpha g_{10}(\alpha)=1$. Note that there is no translation at $\beta=(0,0)$, so $g_{01}(0)$ is unchanged by the lemma's translational change of variables.

The saddle-node set, after changing to polar coordinates $z=r e^{i \theta}$ is the zero set of the function $G: \mathcal{C}^{2} \times \overline{\mathcal{R}^{+}} \times \mathcal{S} \rightarrow \mathcal{C} \times \mathcal{R}$ defined by

$$
G(\alpha, C, r, \theta)=\left\{\begin{array}{c}
F_{(\alpha, C)}\left(r e^{i \theta}, r e^{-i \theta}\right)-r e^{i \theta} \\
1-\operatorname{tr}(D F)+\operatorname{det}(D F)
\end{array}\right.
$$

The complex part of $G$ being zero is the fixed point condition; the real part being zero is the eigenvalue one condition. $D F$ is defined by

$$
D F=\left(\frac{F_{z}}{F_{\bar{z}}} \frac{F_{\bar{z}}}{F_{z}}\right)
$$

This matrix is similar to the Jacobian matrix we would obtain if Eq. (10) were written in cartesian coordinates. In fact it is obtained by standard complexification and a linear change of variables from the cartesian coordinates to the $(z, \bar{z})$ coordinates. Since we use only the determinant and trace of this matrix, the eigenvalue one condition is valid with the matrix in either coordinates. The $(z, \bar{z})$ Jacobian is, however, easier to compute than the cartesian coordinate Jacobian.

Note that for $\alpha=0,(z, C)=(0,0)$ is a root of $G$. That this is the unique root is most easily seen by replacing the eigenvalue one real expression of $G$ with $f_{C}^{\prime}(z)-1$. This replacement is possible since $F_{(0, C)}(z, \bar{z})=f_{C}(z)$ is complex analytic. Now the (complex) implicit function theorem can be applied to $\tilde{G}(z, C) \equiv\left\{\begin{array}{l}f_{C}(z)-z \\ f_{C}^{\prime}(z)-1\end{array}\right.$. Now $\tilde{G}: \mathcal{C}^{2} \rightarrow \mathcal{C}^{2}$ and is complex analytic in both variables. The two-by-two complex Jacobian matrix at the origin turns out to be nonzero because of the assumptions that $a_{0}^{\prime}(0)$ and $a_{2}(0)$ are both nonzero. Thus the theorem is established for $\alpha=0$.

The remainder of the proof is to show that for $\alpha$ small but nonzero, the roots of $G$ are a topological circle in the remaining variables: $\left(r e^{i \theta}, C\right) \in \mathcal{C} \times \mathcal{C}$ 
if $g_{01}(0) \neq 0$. We do this by using the implicit function theorem to solve the fixed-point equation for $C=C(\alpha, r, \theta)$ and then plugging the result into the eigenvalue one equation. Since $G_{1}$ depends complex analytically on $C$, we save much algebra by treating it as a single complex variable rather than as two real variables. Then the hypotheses of the implicit function theorem require the nondegeneracy condition $a_{0}^{\prime}(0) \neq 0$, since $\left.\frac{\partial G_{1}}{\partial C}\right|_{(0,0,0, \theta)}=a_{0}^{\prime}(0)$. Since $\left.\frac{\partial G_{1}}{\partial \alpha}\right|_{(0,0,0, \theta)}=g_{00}(0)$ and $\left.\frac{\partial G_{1}}{\partial r}\right|_{(0,0,0, \theta)}=0$, we can further use the implicit function theorem to obtain $C=C(\alpha, r, \theta)=\frac{-g_{00}(0)}{a_{0}^{\prime}(0)} \alpha+O_{2}\left(\alpha^{2}+r^{2}+\alpha r\right)$. There is $\theta$ dependence only in the $O_{2}$ terms. Moreover, we can use $a_{j}(C)=$ $a_{j}(0)+a_{j}^{\prime}(0) C+O\left(C^{2}\right)$ to obtain $a_{j}(C)=-a_{j}^{\prime}(0) \frac{g_{00}(0)}{a_{0}^{\prime}(0)} \alpha+O_{2}\left(\alpha^{2}+r^{2}+\alpha r\right)$.

We are now ready to attack the eigenvalue one part of $G$. We first note that $D F$ is of the form $D F=\left(\begin{array}{c}1+A \\ B \\ 1+\end{array}\right.$ $\alpha\left(2 g_{20}(\alpha) r e^{i \theta}+g_{11}(\alpha) r e^{-i \theta}+O\left(r^{2}\right)\right)$ and $B=\alpha\left(g_{01}(\alpha)+g_{11}(\alpha) r e^{i \theta}+g_{02}(\alpha) r e^{-i \theta}+\right.$ $\left.O\left(r^{2}\right)\right)$. Recall that we have used the lemma to set $a_{1}(C)+\alpha g_{10}(\alpha)=1$. After substituting for $a_{j}(C)$ as at the end of the previous paragraph, the eigenvalue one equation then "reduces" to

$$
\begin{gathered}
0=1-\operatorname{tr}(D F)+\operatorname{det}(D F)=A \bar{A}-B \bar{B} \\
=4\left|a_{2}(0)\right|^{2} r^{2}-|\alpha|^{2}\left|g_{01}(0)\right|^{2}+O_{3}\left(\alpha r^{2}+r^{3}+\alpha^{2} r+\alpha^{3}\right)
\end{gathered}
$$

If $\left|g_{01}(0)\right| \neq 0$, this is, to lowest order in $r$ and $\alpha$, apparently a cone in $z \times$ $\alpha$ variables (recall $z=r e^{i \theta}$ ), with circular cross sections for $\alpha$ constant and nonzero. (If $\left|g_{01}(\alpha)\right| \equiv 0$ after the translation of the Cardioid cusp normal form lemma, then Eq. (11) has the form $0=4\left|a_{2}(0)\right|^{2} r^{2}+r^{2} O_{1}(r, \alpha)$, so $r=0$ is the only local solution to this equation. Thus no blowup would occur as $\alpha$ is perturbed away from zero. Of course, one would have to perform the translation in order to check this condition.)

As to the nontrivial projection of the circular cross sections to the $C$ plane, this is guaranteed by $\left|a_{2}(0)\right| \neq 0$, which ensures that the fixed point surface (which contains the saddle-node curve) is quadratic near $(z, C)=(0,0)$ and will thus project locally at most four-to-one to the parameter plane.

The computer generated saddle-node curves suggest that the projection is 11: a topological circle with 3 cusps. This is also consistent with the singularity theory unfolding of an elliptic umbillic, of which this saddle-node set is an example [Zeeman 1977].

ProOF of the period-doubling blowup theorem 3.2. We wish to find the roots of $G: \mathcal{C}^{2} \times \overline{\mathcal{R}^{+}} \times \mathcal{S} \rightarrow \mathcal{C} \times \mathcal{R}$ defined by

$$
G(\alpha, C, r, \theta)=\left\{\begin{array}{c}
F_{(\alpha, C)}\left(r e^{i \theta}, r e^{-i \theta}\right)-r e^{i \theta} \\
1+\operatorname{tr}(D F)+\operatorname{det}(D F)
\end{array}\right.
$$


The complex part of $G$ being zero is the fixed-point condition; the real part being zero is the negative one eigenvalue condition. $D F=\left(\frac{F_{z}}{F_{\bar{z}}} \frac{F_{\bar{z}}}{F_{z}}\right)$, as in the previous proof; $\alpha$ can be thought of as either complex or real. We are using polar coordinates in the $z$ plane: $z=r e^{i \theta}$. The use of the normal form means the unique fixed point is at $z=0$ for all values of $C$ and $\alpha$. This greatly simplifies the negative one eigenvalue condition because we only need DF at $z=0$. We quickly (compared to the corresponding equation for the saddle-node blowup theorem) see this condition reduces to

$$
\mid\left(a_{1}(C)+1+\left.\alpha g_{10}(\alpha)\right|^{2}=\alpha^{2}\left|g_{01}(\alpha)\right|^{2} .\right.
$$

This describes a geometric circle in the complex variable $a_{1}$ : center $-1-\alpha g_{10}(\alpha)$ and radius $\left|\alpha g_{01}(\alpha)\right|$. Since our assumptions require $a_{1}^{\prime}(0) \neq 0$, this circle in the $a_{1}$ plane corresponds to a topological circle in the $C$ plane as well. If $\alpha=0$ or $g_{01}(\alpha)=0$, the circle degenerates to a point. This is stronger than the statement of the theorem. Certainly if $g_{01}(0) \neq 0$, then $g_{01}(\alpha) \neq 0$ for $\alpha$ sufficiently small.

ProOF of the bulb contact point blowup theorem 3.3. For any nonzero $\alpha$, the conditions that $\left|g_{21}(0)\right| \neq 0$ and $\left|g_{0, q-1}(0)\right| \neq 0$ are precisely the conditions required for the Arnold tongues to exist [Arnold 1983].

\section{Summary}

This paper presents preliminary results on the problem of bifurcations which evolve when a one-complex-parameter family of maps of the complex plane is perturbed in a non-complex-analytic way. The bifurcation pictures computed for this paper tell a fascinating story, but they are certainly not the complete story. Obviously missing are any curves of global bifurcations - homoclinic tangencies, for example. Detailed studies including these global bifurcations are expected to resemble the detailed studies of Peckham [1988] and Vance and Ross [1989]. These two references are both bifurcation studies of forced oscillator systems, but both include Hopf bifurcation scenarios, and the details near the Hopf bifurcations are similar to the details we would expect to add in Figs. 4 and 5 .

The analytical results presented here still leave unanswered questions. For example, we have not treated the period-two saddle-nodes which appear in Fig. 4d in the period-doubling point blowup. Also the contact point blowup conjecture remains to be proved. Furthermore, we have treated in our theorems only one bifurcation locus at a time. Work in progress [Montaldi and Peckham, in prep.] studies the fixed-point saddle-nodes, period-doublings, and extended Hopf loci as a single unit. There is a surprising amount of complexity added to the resulting bifurcation and singularity problem even when we group the three 
loci together. Including higher period phenomena would further increase the complexity.

We note that all the results we have presented in this paper are local ones. Since the phenomena we studied are all near Hopf bifurcation curves, where the Jacobian derivative matrix is necessarily nonsingular, we have not addressed at all the issue of noninvertibility for these maps. To continue in this direction, we would be studying what the noninvertible map researchers call a " $Z_{2}-Z_{4}$ " map. That is, the number of preimages of points in the plane is either two or four. We are aware of papers of Bielefeld et. al. [1993] and Nien [preprint, 1996] addressing global dynamics of maps which are close to complex analytic. A better understanding of the dynamics of such maps, and the relationship to corresponding complex analytic maps, is still being pursued.

\section{Acknowledgements}

The author wishes to thank J. Drexler, whose master's project work [Dr 1996], under the direction of the author, rekindled the author's interest in this subject. Some of the figures in this paper were suggested by figures in his project. Discussions with J. Montaldi, in the process of preparing a related paper [Montaldi and Peckham, in prep.] were extremely useful. Continuing input and encouragement from D. G. Aronson, P. Blanchard, R. L. Devaney, G. R. Hall, I. G. Kevrekidis, R. P. McGehee, and R. Moeckel are also gratefully acknowledged. Some of their ideas have undoubtedly made their way into this paper.

The author wishes to thank both the Institute NonLineaire de Nice, and the Applied Mathematics Department of the University of Colorado, Boulder for hospitality and computer support provided while work for this paper was being done.

This research was partially supported by NSF Grant DMS-9505051. 


\section{Figure Captions}

1. Approximation of the Mandelbrot set.

2. Some numerically computed bulb boundaries of which the Mandelbrot set boundary is comprised $(\alpha=0.0)$.

3. Generic Hopf bifurcation phenomena a) Bulb tangencies in the complex setting b) Arnold tongues in the real setting.

4. Numerically computed $C$ plane bifurcation diagrams for $\alpha=+0.1$. a) all computed curves, b,d,e,g,h) enlargements of (a), c) enlargement of (b), f) enlargement of (e).

5. Enlargement of parameter plane near the cardioid cusp for $\alpha=+0.1$.

6. Fixed-point phase portraits near the cardioid cusp.

7. Fixed and period-two point phase portraits near the period doubling point.

8. Fixed and period-five point phase portraits near period-5 2/5 resonant Hopf point.

9. Numerically computed saddle-node loci for $\alpha=-0.1$, projected to the $z$ phase plane a) $2 / 5$ saddle-nodes, b) $1 / 3$ saddle-nodes. 


\section{References}

Arnold, V.I. [1983] Geometrical Methods in the Theory of Ordinary Differential Equations, (Springer Verlag, New York).

Blanchard P. [1984], "Complex analytic dynamics on the Riemann sphere," Bull. Amer. Math. Soc., 11 85-141.

Bielefeld B., Sutherland S., Tangerman F., Veerman J.J.P. [1993], "Dynamics of Certain Nonconformal Degree-Two Maps of the Plane," Experimental Mathematics 2 No. 4, 281-300.

Bogdanov R.I. [1976], "Versal deformation of a singularity of a vector field in the plane in the case of zero eigenvalues," Trudy Seminara Imeni I. G. Petrovskogo, Vol. 2, 23-36, 1976. English translation: Selecta Math. Sovietica, Vol. 1, No. 4, 389-421, 1981.

Devaney R. L. [1989], An introduction to Chaotic Dynamical Systems, (AddisonWesley, New York).

Douady A. and Hubbard C. [1982], "Iteration des polynomes quadratiques complexes," C.R. Acad. Sci. Paris 294, 123-126.

Drexler J. [1966], "A Nonanalytic Perturbation of the Complex Quadratic Family of Maps," Master's Project University of Minnesota, Duluth, Technical Report 96-5.

Guckenheimer J and Holmes P [1983] Nonlinear Oscillations, Dynamical Systems and Bifurcations of Vector Fields, Applied Mathematical Sciences, 42 (Springer Verlag, New York).

McGehee R.P. \& Peckham B.B. [1994], "Resonance Surfaces for Forced Oscillators," Geometry Center Research Report GCG70 and Experimental Mathematics 3(3), 221-244.

Montaldi J. and Peckham B.B. [in preparation], "Real Perturbation of the complex quadratic familiy: Fixed point bifurcation loci."

Nien C-H [preprint], "The Dynamics of Planar Quadratic Maps with Nonempty Bounded Critical Set."

Peckham B.B. [1988], "The Closing of Resonance Horns for Periodically Forced Oscillators," Ph. D. thesis, University of Minnesota. 
Peckham, B.B. [1990], "The Necessity of the Hopf Bifurcation for Periodically Forced oscillators with closed resonance regions," Nonlinearity (3), 261-280.

Peckham, B.B. [1988-97], To Be Continued ..., a continuation software package for discrete dynamical systems (continually under development).

Peckham B.B. and Kevrekidis I.G. [1991], "Period doubling with higher order degeneracies" SIAM J. Math. Anal., Vol. 22, No. 6, 1552-1574.

Ruelle D. [1989], Elements of Differentiable Dynamics and Bifurcation Theory, (Academic Press, Inc., San Diego).

Takens F. [1974], "Forced oscillations and bifurcations," Applications of Global Analysis, Communications of the Mathematical Institute Rijksuniversiteit Utrecht, Vol. 3, 1-59.

Vance W. and Ross J. [1989], "A detailed study of a forced chemical oscillator: Arnold tongues and bifurcation sets," J. Chem. Phys. 91, 7654-7670.

Zeeman E.C. [1977], Catastrophe Theory: Selected papers 1972-77, AddisonWesley. 